\title{
Vertical Ionization Energies and Electron Affinities of Native and Damaged DNA Bases, Nucleotides, and Pairs from Density Functional Theory Calculations: Model Assessment and Implications for DNA Damage Recognition and Repair
}

\author{
Polydefkis Diamantis, ${ }^{(\circledR)}$ Ivano Tavernelli, ${ }^{\dagger}{ }^{\circ}$ and Ursula Rothlisberger*(i) \\ Laboratory of Computational Chemistry and Biochemistry, École Polytechnique Fédérale de Lausanne, Swiss Federal Institute of \\ Technology, CH-1015 Lausanne, Switzerland
}

Supporting Information

\begin{abstract}
To assess the effect of an 8-oxoguanine (8OG) defect base on the vertical ionization energies (VIEs) and electron affinities (VEAs) of DNA, density functional theory calculations were carried out for native and defect DNA bases and nucleotides, as well as for larger fragments containing one or multiple pairs. Absolute values of VIE and VEA under implicit solvation did not converge as a function of model size even up to the largest systems taken into consideration ( 3 base pairs/ 2 nucleotide pairs). Nonetheless, a consistent trend was observed for the relative difference in the VIE of native and damaged DNA showing that the defect was lowering the VIE by $-0.1 \mathrm{eV}$ for the largest fragments. This strongly suggests that the presence of $8 \mathrm{OG}$ makes the DNA more easily oxidizable and is in line with experimental evidence that a defect region can act as a sink of oxidative damage. In contrast, relative differences in VEA were very small and varied inconsistently around $0.01 \mathrm{eV}$. This seems to indicate that insertion of $8 \mathrm{OG}$ has a negligible effect on the electron capturing properties of DNA. Similar conclusions can be

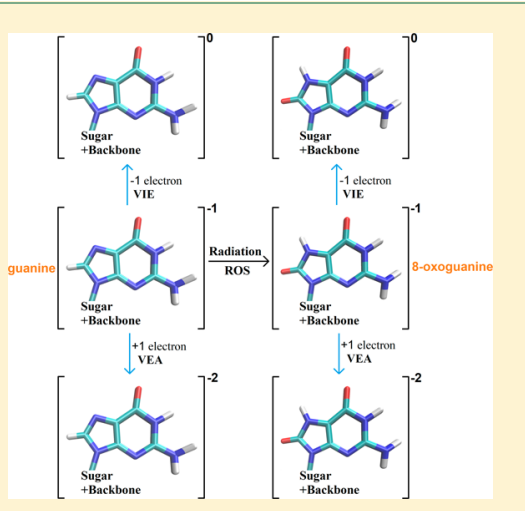
drawn by the adiabatic IEs and EAs computed for some of the larger fragments. Analysis of the hole and excess electron distributions was consistent with the above trends. The findings presented here support the possibility that a mechanism based on hole transport through DNA may be efficiently employed by the cell for the detection of defect bases.
\end{abstract}

\section{INTRODUCTION}

The DNA of living organisms is exposed to various potentially harmful agents, ranging from radiation to reactive oxygen species (ROS), which can cause different types of damage to its molecular building blocks. Depending on the type of lesion, cells have evolved a series of complex, yet highly efficient repair pathways in order to preserve genome integrity. ${ }^{1}$ Among these pathways, the Base Excision Repair (BER) ${ }^{2,3}$ pathway is of special importance, as it deals with the repair of the vast majority of DNA base lesions, in particular also those that are somewhat elusive since they do not distort the DNA's double helical structure. Recognition of DNA lesions is of prime importance as they are often associated with the development of diseases such as neurodegenerative disorders and cancer. ${ }^{3}$ The molecular agents that are involved in detecting and eventually removing these defects are the BER enzymes. ${ }^{4}$ Despite the fact that the structure of many of these enzymes is well-known and their specific biological action has been established, there is still inconclusive evidence on how they coordinate their function in order to have the full genome examined within a reasonable amount of time with respect to typical cell life times.

A promising hypothesis has recently been proposed by Barton and co-workers. ${ }^{5-10}$ The proposed mechanism is based on the possibility that two BER enzymes cooperate together to detect the position of a defect in double stranded (ds) DNA. The process is initiated by the presence of an oxidative agent (e.g., a guanine radical), which is repaired by an enzyme that binds to the DNA and is in turn oxidized. Following this action, a second BER enzyme that attaches to the DNA in proximity of the first enzyme can inject an electron. This electron travels through the sequence and, in the absence of lesions, is ultimately absorbed by the first enzyme, which then detaches and binds to another position on the DNA. In contrast, if the DNA defect acts as a sink that traps the electron, the two enzymes remain bound to the DNA, and the excess electron transfer step cannot be completed. The first BER enzyme, which is still in its oxidized state, diffuses to the defect, eventually repairs it, and subsequently detaches from the DNA. The ability of DNA to capture excess electrons has been demonstrated for both native ${ }^{11,12}$ and damaged ${ }^{12}$ sequences. Furthermore, the ability of DNA to conduct holes and excess electrons is well established. ${ }^{13}$ It has been suggested that in Escherichia coli, an electron transfer length of 200 to 500 base pairs between two BER enzymes is sufficient for rapidly inspecting the full genome in a time well within the cell's doubling time. ${ }^{6}$ A charge transfer recognition mechanism as

Received: June 26, 2018

Published: January 25, 2019 
suggested by Barton is also supported by the theoretical study of Eriksen, ${ }^{14}$ who concluded that, in comparison with models based on independently acting BER enzymes, a tandem recognition mechanism could reduce the time needed to fully scan E. coli's genome by roughly an order of magnitude.

In addition, both $\mathrm{G}$-rich ${ }^{15}$ and 8-oxoguanine (8OG)containing $^{16,17}$ double stranded DNA fragments have been proposed to have low ionization energies (lower than those of single $G, A, C$, and $T$ bases) that allow them to act as sacrificial hole traps, by "filtering" oxidative damage, and thus preventing the rest of the DNA from further assault.

In view of all this, a comprehensive knowledge of the redox properties of native and damaged DNA under physiological cellular conditions is essential in order to understand and evaluate possible implications for defect recognition and repair. In particular, the vertical electron affinities (VEAs) and vertical ionization energies (VIEs) of DNA bases and nucleotides and their sequence dependence could provide valuable information.

During the last years, a relatively clear picture has emerged for VIEs. G is experimentally ${ }^{18-22}$ and computationally ${ }^{22-25}$ known to have the lowest VIE among the four bases in the gas phase. Experimental estimates lie in the range of 8.0-8.3 eV. ${ }^{18-22}$ Moreover, recent studies by Jungwirth et al. ${ }^{26-28}$ have demonstrated that, albeit in gas phase, a great dependence of VIE on the size of the building block exists, this sensitivity is drastically reduced under aqueous solution conditions. For instance, VIEs determined with Møller-Plesset Perturbation Theory (MP2) for guanine nucleosides and nucleotides and G$\mathrm{C}$ base pairs range from 7.00 to $7.30 \mathrm{eV}^{26}$ when a polarizable continuum model is used to represent the solvent, in good agreement with experimental estimates for the VIE of the G base and nucleotide in aqueous solution of the order of 7.0 $\mathrm{eV}^{28}$ Concerning larger fragments, calculations for stacks of two G bases with DFT ${ }^{25}$ and for stacks of up to four G bases and two G-C pairs with Hartree-Fock $(\mathrm{HF})^{29}$ have revealed a tendency of VIEs to decrease when the system size is increased. Similar conclusions were drawn from $\mathrm{HF}^{30}$ and DFT(M06-2X $)^{31}$ calculations covering an even larger range of system sizes (up to $6 \mathrm{G}-\mathrm{C}$ nucleotide pairs, ${ }^{30} 10 \mathrm{G}$ bases, ${ }^{31}$ and 8 G-C base pairs, ${ }^{31}$ respectively). In fact, in gas-phase calculations, no convergence of the VIE values was achieved up to the maximum system sizes considered, ${ }^{30,31}$ and even in calculations with the COSMO solvation model, although VIEs are less size-dependent, ${ }^{30}$ convergence was not completely reached at a system size of 6 nucleotide pairs $(\mathrm{VIE} \approx 5.70 \mathrm{eV})$.

The situation is quite different in the case of EAs, ${ }^{32,33}$ where there is still no conclusive evidence concerning the exact values for nucleobases neither from experimental ${ }^{34-38}$ nor from computational $^{23,32,33,39-48}$ studies. All experimental values available so far have been determined in the gas phase, and it is not clear to what extent the gas phase data are representative for the biological, condensed phase environment. The majority of the published computed values (at HF, DFT, or MP2 level) also correspond to the gas phase, and in agreement with experiments, report negative EA values, indicating unstable anions. Recently, Pal et $\mathrm{al}^{33}$ reported negative VEAs for DNA nucleobases in the gas phase computed with an Equations of motion/Coupled cluster scheme (EOM-CCSD) providing values closer to the experimental range than previous efforts. For guanine, the computed VEA is $-0.23 \mathrm{eV}$, compared to an experimental estimate of $-0.46 \mathrm{eV}$ for the enol form. ${ }^{34}$ For thymine, cytosine, and adenine, the computed VEAs by Pal et al. $(-0.28$ $\mathrm{eV},-0.27 \mathrm{eV}$, and $-0.44 \mathrm{eV}$, respectively) are very close to the highest experimental estimates $(-0.29 \mathrm{eV},-0.32 \mathrm{eV}$, and $-0.45 \mathrm{eV}$, respectively). The negative gas phase values reported from both experiments and simulations indicate that, while energetically unfavorable, electron attachment can lead to kinetically stable "vertical" anions that momentarily appear as resonant states, as already discussed in the context of both theoretical ${ }^{33}$ and experimental ${ }^{34}$ studies. Accurate measurements of gas phase VEAs for the nucleobases are further complicated through the presence of interchanging tautomers that are very close in energy. This phenomenon is especially pronounced in guanine for its keto and enol forms, ${ }^{34}$ with their relative energy difference computed to be of the order of only $0.08 \mathrm{kcal} / \mathrm{mol}^{33}$

On the other hand, it has been suggested that electron attachment is favored in condensed phase environments due to the positive solvation free energies of bases and base pairs (of the order of 2 to $3 \mathrm{eV})^{49,50}$ estimated from microhydration models of DNA building blocks, leading to positive adiabatic electron affinities. However, it is also necessary to establish the dependence of the electron affinity values as a function of model size. So far, a possible size dependence has only been tested up to the level of single pairs or three-base stacks $^{48,49,25,51-56}$ revealing VEAs significantly larger than for single bases, thus displaying a similar size dependence as in the case of gas phase VIEs.

In the present work, we aim at gaining more insight into the effects of a lesion on DNA's electron and hole capturing properties. In particular, we focus on guanine (G) and its highly common lesion, 8-oxoguanine (8OG).

On the basis of low energy photoelectron transmission (LEPET) and two photon photoemission (TPPE) spectroscopy experiments, it has been suggested that, due to a higher density of unoccupied electronic states, 8OG-containing fragments are more capable of capturing excess electrons than their G-containing counterparts. ${ }^{12} \mathrm{Up}$ to date, computed VIE values of $80 G$ under implicit solvation conditions are available only at the single base and nucleotide level, amounting to 6.94 and $6.79 \mathrm{eV}$, respectively. ${ }^{57}$ Moreover, two calculated values for the VIE of an 8 OG base in the gas phase are also available and amount to $7.84 \mathrm{eV}^{57}$ and 7.87 $\mathrm{eV} .{ }^{58}$ In addition, a numerical value for the VEA of the same system and conditions has also been reported as $-0.22 \mathrm{eV} .^{59}$

To our knowledge, unlike native DNA, no systematic study of VIEs/VEAs as a function of system size has been carried out for defect (8OG-containing) DNA fragments. Such an investigation, and comparison with the respective native fragments-especially in aqueous solution-is essential for making an assessment of 8OG's effect on the redox properties. To this end, we calculate here the vertical electron affinities and ionization energies of native and damaged DNA fragments both in the gas phase and under implicit solvation conditions. Model systems of varying size have been considered (ranging from single bases/nucleotides to base/nucleotide pair stacks). In order to assess whether the findings from the VIE/VEA calculations still apply to relaxed geometries, adiabatic ionization energies and electron affinities (AIEs/AEAs) for some of the larger fragments have also been calculated. Finally, aiming to gain a better understanding of the difference in charge capturing properties between native and defect DNA, the distribution of the hole and the excess electron following 
Table 1. DNA Bases, Nucleotides, and DNA Fragments under Study and the Nomenclature Used To Refer to the Different Models $^{a}$

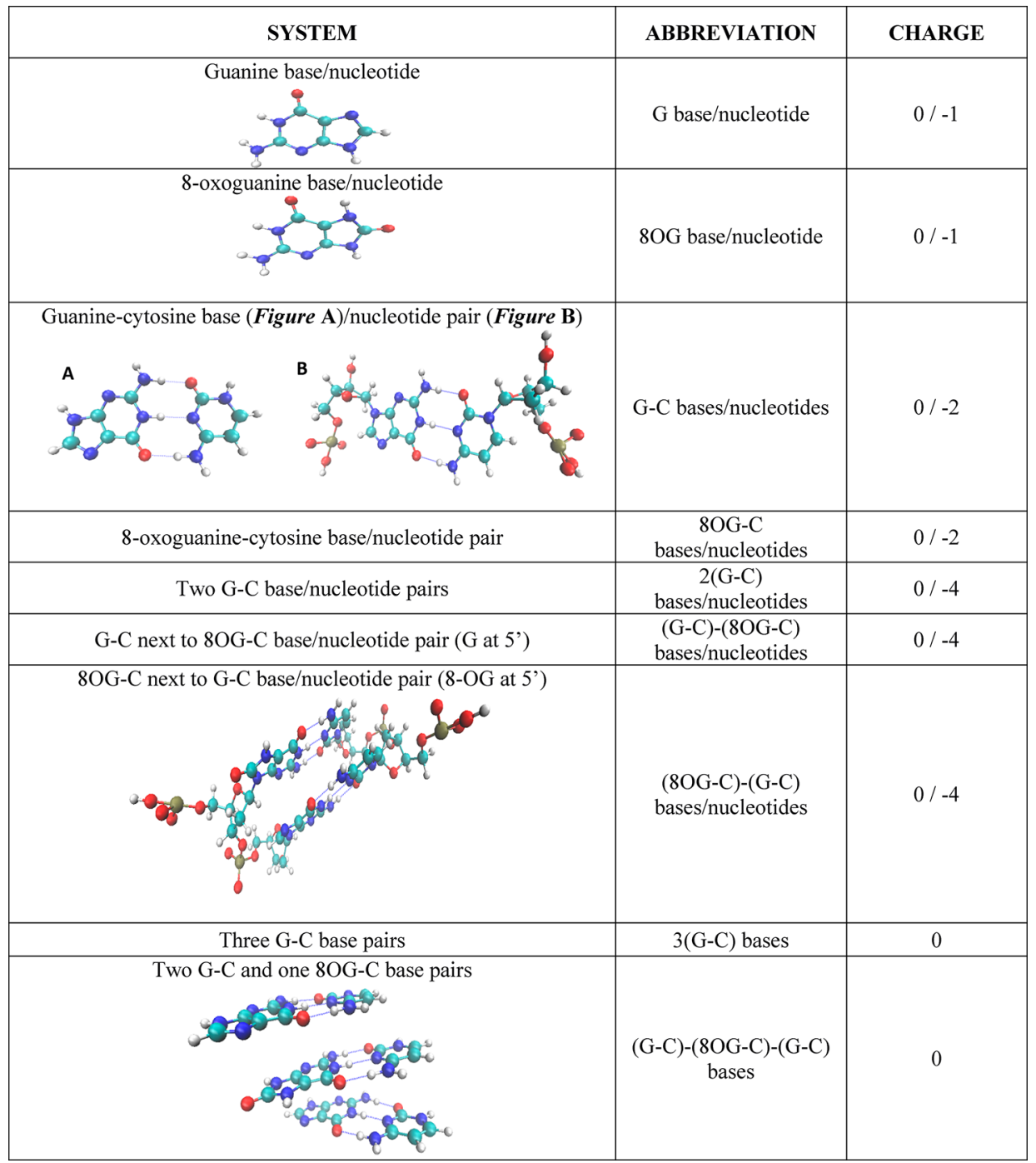

${ }^{a_{T}}$ The charge of each system is also given. Snapshots of some systems have also been included.

ionization and electron attachment, respectively, are also analyzed.

\section{COMPUTATIONAL METHODS}

All calculations were performed with the Gaussian 09 package $^{60}$ using density functional theory. ${ }^{61,62}$ As previously stated, calculations of the vertical energy gaps were performed both in the gas phase and under implicit solvation applying a Polarizable Continuum Model ( $\mathrm{PCM}^{63-65}$ ) for water. In order to assess the performance of different basis sets and functionals for the calculation of VIEs/VEAs, G and 8OG bases/ nucleotides were described at the $\operatorname{BLYP}^{66,67} / 6-31+\mathrm{G}(3 \mathrm{df}, 2 \mathrm{p})$, BLYP/TZVP, ${ }^{68}$ B3LYP $^{69,67} / 6-31+\mathrm{G}(3 \mathrm{df}, 2 \mathrm{p})$, B3LYP/6-31+ $+\mathrm{G}^{*},{ }^{71-76} \mathrm{M}^{70} / 6-31++\mathrm{G}^{*}$, and M06/TZVP levels of theory. Recently, the VIE of the guanine base was evaluated by Sevilla et al. ${ }^{77}$ with the $\omega \mathrm{B} 97 \mathrm{X}$ functional ${ }^{78}$ yielding a value of $8.14 \mathrm{eV}$. To assess the overall performance of this functional, calculations were performed with $\omega \mathrm{B} 97 \mathrm{X}$ and its $\omega \mathrm{B} 97 \mathrm{XD}$ variant $^{79}$ in combination with a $6-31++G^{*}$ basis set for the building blocks for which experimental data are available (VIE of $G$ base and nucleotide, in gas phase and in aqueous solution). Since we aim at calculating vertical energy gaps, the geometry of each system was optimized only in the initial oxidation state.

In the calculations of VIEs and VEAs with the PCM model, nonequilibrium solvation effects have to be considered. ${ }^{80,81}$ In particular, the response of the solvent to a change of the solute's charge can be divided into a fast component accounting for the electronic response and a slow component accounting for the nuclear response. If both oxidation states are treated with a standard PCM protocol, the solvent's nuclear response is also included. Therefore, the calculated energy difference between the two states does not correspond to a vertical energy gap. To account for this, we have used the nonequilibrium PCM (NEPCM) model $^{80,81}$ as implemented in Gaussian 09. The NEPCM protocol consists of an optimization of the initial state with the standard PCM model, a subsequent self-consistent field (SCF) step at the optimized geometry while saving information for both response components, and the calculation of the solute's energy in the final state while considering only the electronic response of the solvent. The NEPCM model has been 
successfully employed by Jungwirth and co-workers for the determination of the vertical ionization energies of neutral ${ }^{82}$ and protonated ${ }^{83}$ imidazole, as well as of DNA building blocks. ${ }^{26-28,84}$

In the case of the nucleotide systems, only the anticonformation has been considered as it is the relevant form that is adopted within the DNA environment. The 5' and 3' open valencies were capped with an $-\mathrm{OH}$ group and an $-\mathrm{H}$, respectively, yielding a total charge of -1 for each nucleotide.

Given that accurate VIEs of small charged DNA building blocks can be accurately reproduced even in the absence of counterions both in gas phase and with the NEPCM protocol, ${ }^{26,27,57}$ counterions were not included in the present study. Test calculations were nonetheless performed for both $\mathrm{G}$ and $8 \mathrm{OG}$ bases and nucleotides with a $\mathrm{Na}^{+}$counterion, both in gas phase and with the PCM model at the B3LYP/6-31+ $+\mathrm{G}^{*}$ level of theory in order to assess possible effects on VIE and VEA. These calculations are reported in the Supporting Information.

For systems in which AIEs/AEAs were also calculated, their geometry was also optimized in their reduced and oxidized states.

All DNA bases and fragments investigated in this work are presented in Table 1 together with the abbreviations used for the different models and representative snapshots. All optimized coordinates of the neutral species (nucleotide charge equal to -1 ), as well as the optimized reduced and oxidized geometries in the case of AIEs/AEAs, are available in the Supporting Information.

\section{RESULTS AND DISCUSSION}

Based on the performance of all combinations of functionals and basis sets for bases and nucleotides with respect to (i) experimental and theoretical values available $e^{18-59,85}$ and (ii) convergence issues (discussed in detail in the Supporting Information), all the calculations presented below have been carried out at the B3LYP/6-31++G* level of theory. Moreover, given that the outcome of calculations performed with the PCM model is of greater biological significance, VIEs and VEAs of gas phase systems are only given in the Supporting Information.

G and $80 \mathrm{G}$ Bases and Their Nucleotides. The B3LYP/ 6-31++G*-computed vertical energy gaps of $G, 8 \mathrm{OG}$, and their nucleotides under implicit solvation conditions are reported in Table 3. VIEs and VEAs computed with other DFT functional/basis set combinations are available in Table S2 of the Supporting Information.

All VEA values obtained with the PCM model (Table 2 and Table S2 in the Supporting Information) display a very strong dependence on the basis set, both for bases and for nucleotides. Insertion of the sugar and phosphate moieties has a weak effect on the PCM-computed VEAs. For the values

Table 2. Computed VEAs and VIEs of G and 8OG and Their Nucleotides with the Polarizable Continuum Model at Geometries Optimized at the B3LYP/6-31++G* Level of Theory for the Neutral Systems

\begin{tabular}{|c|c|c|c|c|c|c|c|}
\hline \multicolumn{2}{|c|}{$\mathrm{G}$ base } & \multicolumn{2}{|c|}{ G nucleotide } & \multicolumn{2}{|c|}{$80 G$ base } & \multicolumn{2}{|c|}{$80 G$ nucleotide } \\
\hline $\begin{array}{l}\text { VEA } \\
(\mathrm{eV})\end{array}$ & $\begin{array}{l}\text { VIE } \\
(\mathrm{eV})\end{array}$ & $\begin{array}{l}\text { VEA } \\
(\mathrm{eV})\end{array}$ & $\begin{array}{l}\text { VIE } \\
(\mathrm{eV})\end{array}$ & $\begin{array}{l}\text { VEA } \\
(\mathrm{eV})\end{array}$ & $\begin{array}{l}\text { VIE } \\
(\mathrm{eV})\end{array}$ & $\begin{array}{l}\text { VEA } \\
(\mathrm{eV})\end{array}$ & $\begin{array}{l}\text { VIE } \\
(\mathrm{eV})\end{array}$ \\
\hline 0.01 & 7.19 & 0.07 & 7.08 & 0.08 & 6.89 & 0.14 & 6.82 \\
\hline
\end{tabular}

reported in the SI (Table S2), the presence of a stabilizing aqueous environment still leads to negative VEA values, in contrast with previous suggestions. ${ }^{49,50}$ Even with B3LYP/6$31++G^{*}$ (Table 2), all PCM-computed VEAs are just slightly positive. A positive VEA for $\mathrm{G}(1.01 \mathrm{eV})$ computed at the B3LYP/D95 V+(D) level of theory has previously been reported, ${ }^{42}$ and the disagreement with the values obtained in the present work might be attributed to the use of different DFT functionals and basis sets and the calculation of VEA on a gas phase-optimized geometry, as well as the absence of a NEPCM protocol.

Despite a lack of reference values in aqueous solution, the electron capturing ability of $\mathrm{G}$ and $8 \mathrm{OG}$ treated at the same level of theory can be compared, since essentially all employed methods and basis sets result in the same qualitative trends, indicating that the oxidized guanine base $8 \mathrm{OG}$ has a higher VEA than its native counterpart under implicit solvation, and the same holds for the case of the corresponding nucleotides. Such a finding is in support of the CT-based model for the detection of DNA defects proposed by Barton and co-workers, in which $8 \mathrm{OG}$ is a stronger electron trap than $\mathrm{G}$, and may thus prevent the completion of the CT step between the two BER enzymes.

Regarding VIEs, addition of the sugar group and the phosphate backbone leads to just a small change of VIE in PCM, in agreement with previous investigations. ${ }^{26-28}$ The B3LYP values for G base and nucleotide are in very good agreement with the experimental estimate for both types of fragments $\left(7.00 \pm 0.20 \mathrm{eV}^{28}\right)$. It has been stated ${ }^{16,17}$ and shown computationally ${ }^{55,56}$ that the defect base $8 \mathrm{OG}$ is more highly prone to oxidation than G and possesses a lower VIE than the native base. The ionization energies computed in the present work (Table 2 and Table S2 in the Supporting Information) are also in line with these findings, with the VIE of the $8 \mathrm{OG}$ base/nucleotide being $0.20-0.35 \mathrm{eV}$ lower than the one of the respective native system.

Fragments with One or Multiple Base/Nucleotide Pairs. The above calculations on single $\mathrm{G}$ bases/nucleotides with the PCM model show that with the inclusion of sugar and phosphate groups to the base only minor changes are observed, suggesting that this model size is sufficient to ensure convergence to DNA VIE and VEA in physiological conditions. However, the inclusion of the complementary bases/nucleotides and the addition of 2 more base/1 more nucleotide pairs (Tables 3 and 4) show that convergence is actually still not reached, and considering even larger DNA fragments is necessary.

In Tables 3 and 4, the vertical energy gaps of the base pairs (Table 3) and the nucleotide pairs (Table 4), computed employing the B3LYP functional, a $6-31++\mathrm{G}^{*}$ basis set, and the polarizable continuum model, are presented.

The inclusion of cytosine as a hydrogen-bonding partner lowers the ionization energy and enhances the electron capturing ability of G-C/8OG-C base pairs with respect to single $G$ and $80 G$ bases (Tables 3 and 4). For single nucleotide pairs, both VIEs and VEAs are very weakly sensitive to the addition of the phosphate and sugar. VIEs decrease by around $0.05 \mathrm{eV}$, while VEAs increase by around $0.1 \mathrm{eV}$, respectively, upon backbone inclusion.

Table 3 demonstrates that both VIEs and VEAs are weakly affected by the increase in the number of base pairs, especially in the case of the VEA. VIEs decrease by $0.50 \mathrm{eV}$, while VEAs are augmented by $0.36 \mathrm{eV}$ when increasing the number of pairs 
Table 3. Computed VEAs and VIEs of Base Pairs and Small Native and Damaged Base Pair Stacks under Implicit Solvation (PCM water), at the B3LYP/M06-6-31++G* Level of Theory ${ }^{a}$

\begin{tabular}{lcc}
\multicolumn{1}{c}{ system } & VEA $(\mathrm{eV})$ & VIE $(\mathrm{eV})$ \\
G-C bases & 0.49 & 6.90 \\
8OG-C bases & 0.52 & 6.69 \\
2 (G-C) bases & 0.74 & 6.61 \\
(G-C)-(8OG-C) bases & 0.74 & 6.50 \\
(8OG-C)-(G-C) bases & 0.75 & 6.49 \\
3 (G-C) bases & 0.83 & 6.48 \\
(G-C)-(8OG-C)-(G-C) bases & 0.85 & 6.40
\end{tabular}

${ }^{a}$ For fragments composed of three base pairs, VIEs and VEAs are given for both optimization schemes mentioned in the text: protocol (i) (left) and (ii) (right).

Table 4. Computed VEAs and VIEs of Nucleotide Pairs and Small Native and Damaged DNA Fragments (B3LYP functional) under Implicit Solvation (PCM water)

\begin{tabular}{lcc}
\multicolumn{1}{c}{ system } & VEA $(\mathrm{eV})$ & VIE $(\mathrm{eV})$ \\
G-C nucleotides & 0.51 & 6.82 \\
8OG-C nucleotides & 0.48 & 6.65 \\
2 (G-C) nucleotides & 0.61 & 6.38 \\
(G-C)-(8OG-C) nucleotides & 0.67 & 6.29 \\
(8OG-C)-(G-C) nucleotides & 0.63 & 6.34
\end{tabular}

from one to three. Relative VIE differences for fragments composed of 2 and 3 base pairs are 0.12 and $0.08 \mathrm{eV}$, respectively. Although these differences are less pronounced compared to the case of one base pair $(0.21 \mathrm{eV})$, they still demonstrate that the replacement of a $G$ by $80 G$ facilitates DNA oxidation. On the contrary, the same G $\rightarrow$ 8OG replacement appears to have a very weak effect on excess electron capturing, as VEAs of native and defect fragments of the same size are extremely close (with relative differences of the order of $0.01 \mathrm{eV}$ ).

Table 4 suggests the situation does not change significantly upon addition of the sugar and backbone moieties. When moving from one to two nucleotide pairs, VIEs and VEAs decrease and increase, respectively. Relative differences for VIE are slightly higher than for VEA. Similar to base pair systems, the presence of $8 \mathrm{OG}$ decreases the ionization potential. The relative difference in vertical electron affinity is within $0.04 \mathrm{eV}$ in favor of the defect fragments, suggesting a subtle improvement in electron capturing efficiency compared to native DNA.

Overall, the VIE trends observed for G-C base/nucleotide stacks are in line with observations from computational studies on G-C stacks, ${ }^{30}$ while a similar behavior is also observed for the VIE of defect DNA stacks. Contrary to VIEs, VEAs increase as the size of the system increases.

To visually illustrate the trends discussed above, the VIEs and VEAs obtained under implicit solvation are reported in Figures 1 and 2 as a function of system size, distinguishing between base and nucleotide systems. For defect systems composed of 2 base/nucleotide pairs, the VIE/VEA shown in the graphs corresponds to the average of the VIEs/VEAs of the (G-C)-(8OG-C) and (8OG-C)-(G-C) fragments.

Overall, it turns out that, even with implicit solvation, the absolute values of VIEs and VEAs are not converged with respect to system size for both base pairs and nucleotide pairs

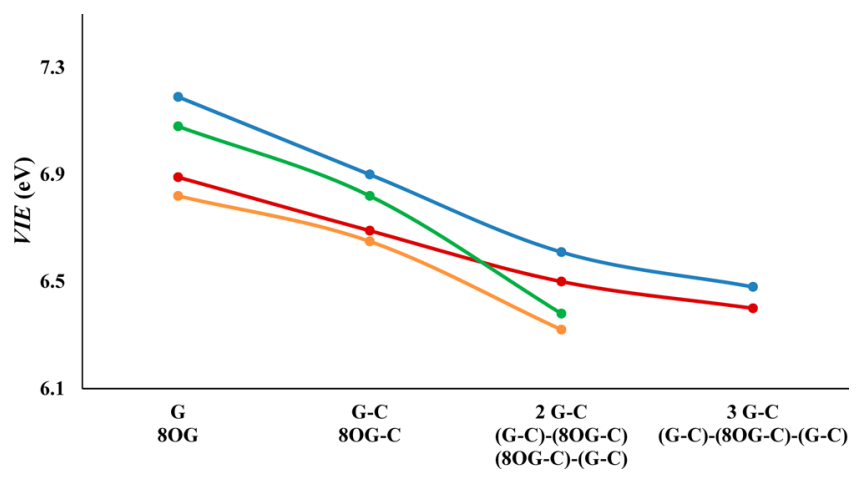

Figure 1. VIEs of native and defect fragments with the polarizable continuum model. Color assignment: native base systems in blue, defect base systems in red, native nucleotide systems in green, and defect nucleotide systems in orange.

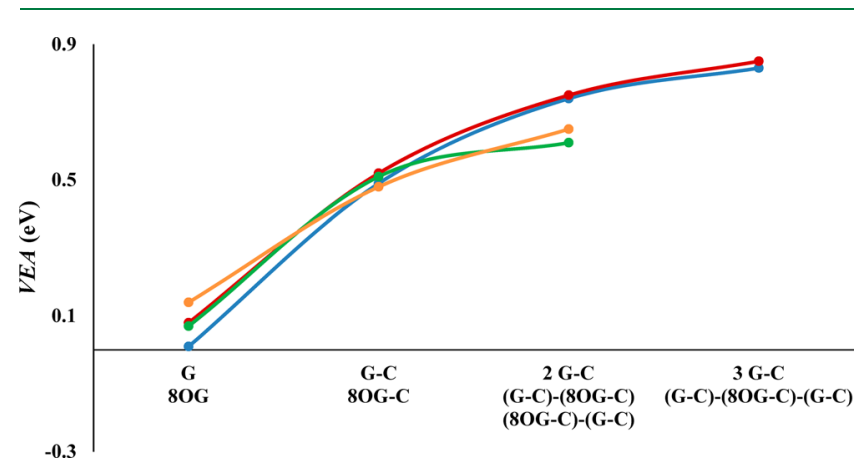

Figure 2. VEAs of native and defect fragments with the polarizable continuum model. Color assignment: native base systems in blue, defect base systems in red, native nucleotide systems in green, and defect nucleotide systems in orange.

even for systems containing up to 3 base/nucleotide pairs, and the examination of even larger fragments in a more realistic (atomistic) environment (e.g., in the presence of explicit counterions and solvent molecules) might be necessary.

On the other hand, a comparison of native and 8OGcontaining base/nucleotide stacks over the entire spectrum of system sizes considered (Figure 1) reveals a consistent trend up to the largest fragments. The VIE of defect fragments is around $0.1 \mathrm{eV}$ lower than their native counterparts. This finding is in line with the notion that $80 G$ renders DNA more easily oxidizable and the suggestion that $8 \mathrm{OG}$ acts as a hole trap. On the contrary, the situation is less clear for VEAs (Figure 2); with smaller and inconsistent differences between native and defect fragments (in the range of $0.01 \mathrm{eV}$ ) based on these results, it is very difficult to assess whether 8OGcontaining sites can act as electron traps.

Adiabatic Energy Gaps of Base Stacks. The vertical energy gaps presented in Tables 3 and 4 provide valuable information on the effect of $8 \mathrm{OG}$ on the vertical IEs and EAs of DNA fragments. Within the DNA environment, these vertical states are, however, short-lived, and oxidized or reduced fragments undergo rapid geometric relaxation. It is therefore important to assess whether the conclusions drawn for the vertical states of the DNA fragments also apply to their relaxed, adiabatic states. In Table 5, the adiabatic IEs and EAs of the stacks composed of one and two base pairs are shown.

Table 5 reveals that, upon geometry relaxation, the effect of $8 \mathrm{OG}$ becomes even more pronounced, as the relative AIE difference between native and defect fragments reaches 0.23 
Table 5. Computed AEAs and AIEs of Nucleotide Pairs and Small Native and Damaged DNA Fragments (B3LYP functional) under Implicit Solvation (PCM water)

\begin{tabular}{lcc}
\multicolumn{1}{c}{ system } & AEA $(\mathrm{eV})$ & AIE $(\mathrm{eV})$ \\
G-C bases & 1.95 & 5.61 \\
8OG-C bases & 1.96 & 5.38 \\
2(G-C) bases & 1.96 & 5.59 \\
(G-C)-(8OG-C) bases & 1.96 & 5.36 \\
(8OG-C)-(G-C) bases & 1.97 & 5.36 \\
\hline
\end{tabular}

$\mathrm{eV}$, compared to $0.10 \mathrm{eV}$ for VIEs. Similar to VEAs, AEAs of native and defect fragments are very close, differing by only $0.01 \mathrm{eV}$, further indicating that $8 \mathrm{OG}$ has a very weak effect on the electron capturing properties of the DNA fragments. Compared to vertical gaps, AIEs and AEAs converge sooner with respect to system size, differing by only $0.01 \mathrm{eV}$ for the larger systems. Most importantly, regarding the impact of $8 \mathrm{OG}$ on ionization and electron attachment, the conclusions reached by adiabatic IEs and EAs are the same as those from vertical gaps, indicating that the latter are sufficient for the purpose of this investigation.

Hole and Excess Electron Distributions. Aiming to gain further insights into the differences in electronic properties of native and damaged DNA fragments, we analyzed the localization of the excess electron/hole upon vertical and adiabatic electron attachment/ionization. For each system, this was realized by taking the difference in spin densities before and after hole/excess electron injection. In the case of nucleotide pairs, each of the nucleotides was split in its components (phosphate group, sugar moiety, and guanine/8oxoguanine or cytosine base, respectively). In Figures $3-5$, the

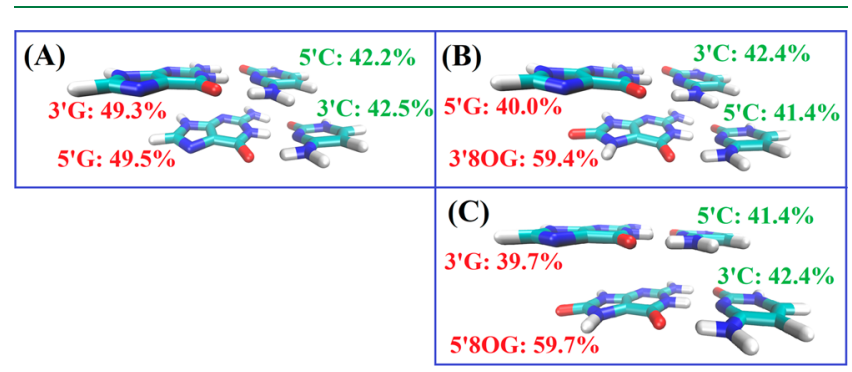

Figure 3. Optimized geometry of the (A) 2 G-C base pairs, the (B) $(\mathrm{G}-\mathrm{C})-(8 \mathrm{OG}-\mathrm{C})$ base pairs, and the $(\mathrm{C})(8 \mathrm{OG}-\mathrm{C})-(\mathrm{G}-\mathrm{C})$ pairs, indicating the system components that concentrate the major fractions of the hole (red) and the excess electron (green). The distributions correspond to vertical IEs/EAs.

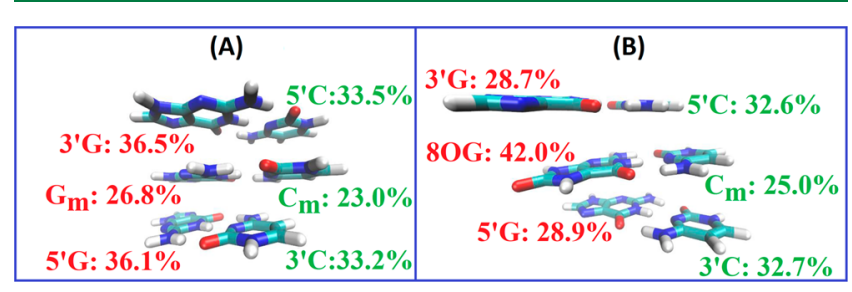

Figure 4. Optimized geometry of the (A) 3 G-C base pairs and the (B) (G-C)-(8OG-C)-(G-C) base pairs, indicating the system components that concentrate the major fractions of the hole (red) and the excess electron (green). The $\mathrm{G}_{\mathrm{m}}$ and $\mathrm{C}_{\mathrm{m}}$ index corresponds to middle guanine and cytosine, respectively. The distributions correspond to vertical IEs/EAs. optimized geometries of neutral base/nucleotide pair stacks are shown, emphasizing the components concentrating the most significant fractions of the hole and excess charge (up to $\approx 10 \%$ ), following vertical ionization or electron attachment. The complete hole and excess distributions for all these base/ nucleotide pair systems are reported in Tables S5-S16 of the Supporting Information, with significant fractions highlighted in bold. The same distributions corresponding to AIEs/AEAs are also available, in Tables S17-S21.

In the G-C/8OG-C base pairs, the hole and the excess electron (Tables S5 and S6 of the Supporting Information) mainly localize on the higher affinity base; i.e., the hole is mainly localized on G or 8OG, respectively (up to $97 \%$ ), while the excess electron resides on C (up to $74 \%$ ), in agreement with the fact that $\mathrm{C}$ has a higher VEA than G. ${ }^{15}$

The hole/excess electron charge distributions corresponding to the G-C and 8OG-C nucleotide pairs are displayed in Tables S12 and S13 of the Supporting Information. The fraction of the hole or the excess electron residing on each of the major components (phosphate, sugar, and base) is explicitly presented. The hole and the excess electron are mainly (up to $88 \% / 73 \%$, respectively) localized on the base of the nucleotide showing the higher affinity (on G-80G/C, respectively). In both cases, a significant fraction of the excess electron charge resides on the respective purine base $(22 \%$ and $31 \%$ on $\mathrm{G}$ and $8 \mathrm{OG}$, respectively).

A comparison of native and defect base pair fragments consisting of 2 and 3 base pairs (Figures 3 and 4, Tables S7S11) reveals that the presence of $8 \mathrm{OG}$ significantly alters the distribution of the hole charge, with a larger fraction of it being found on the defect base. More precisely, in the $2 \mathrm{G}-\mathrm{C}$ pairs (Figure 3A), the hole is evenly shared by the two $\mathrm{G}$ bases (approximately $49.5 \%$ on each of them). On the other hand, the same distributions in the (G-C)-(8OG-C) and (8OG-C)(G-C) pairs (Figure 3B,C) are characterized by $60 \%$ of the hole charge on $8 \mathrm{OG}$, compared to $40 \%$ for G. A similar difference is also observed in the fragments composed of 3 base pairs (Figure 4). In the $3 \mathrm{G}-\mathrm{C}$ pairs (Figure 4A), the hole is distributed between the three guanines (34\%, 23\%, 33\%), while in the defect fragment (Figure 4B), 8OG concentrates $42 \%$ of the hole charge, compared to $29 \%$ for each of the two $\mathrm{G}$ bases. The situation is less clear for the excess electron with very similar charge distribution in the native and defect fragments, thus suggesting that the presence of $80 G$ has a negligible effect on the excess charge distributions.

Overall, the charge distributions of systems composed of two nucleotide pairs (Figure 5A) follow a pattern, which is similar to that of the respective base pair systems. In the $2 \mathrm{G}-\mathrm{C}$ pairs, the hole resides on the two $\mathrm{G}$ bases ( $45 \% 50 \%$ ), while the excess electron is localized on the two $C$ bases $(46 \%, 41 \%)$. The insertion of $8 \mathrm{OG}$ (Figure 5B,C), independently of its position $\left(5^{\prime}\right.$ or $\left.3^{\prime}\right)$, leads to hole distributions that are very similar to those of the base pair systems, with $80 \mathrm{OG}$ concentrating $58 \%$ of the hole charge, followed by $\mathrm{G}$ with $40 \%$. The excess electron distributions for defect nucleotide fragments are also different than the native ones but show an inconsistent trend. In (G-C)-(8G-C), the guanine's complementary $\mathrm{C}$ base concentrates the largest fraction of the excess electron charge $(49 \%)$, followed by the other C $(38 \%)$. The opposite occurs for the (8OG-C)-(G-C) system, with 8 OG's complementary $\mathrm{C}$ base concentrating $53 \%$ of the excess electron charge, compared to $33 \%$ for the other C. 


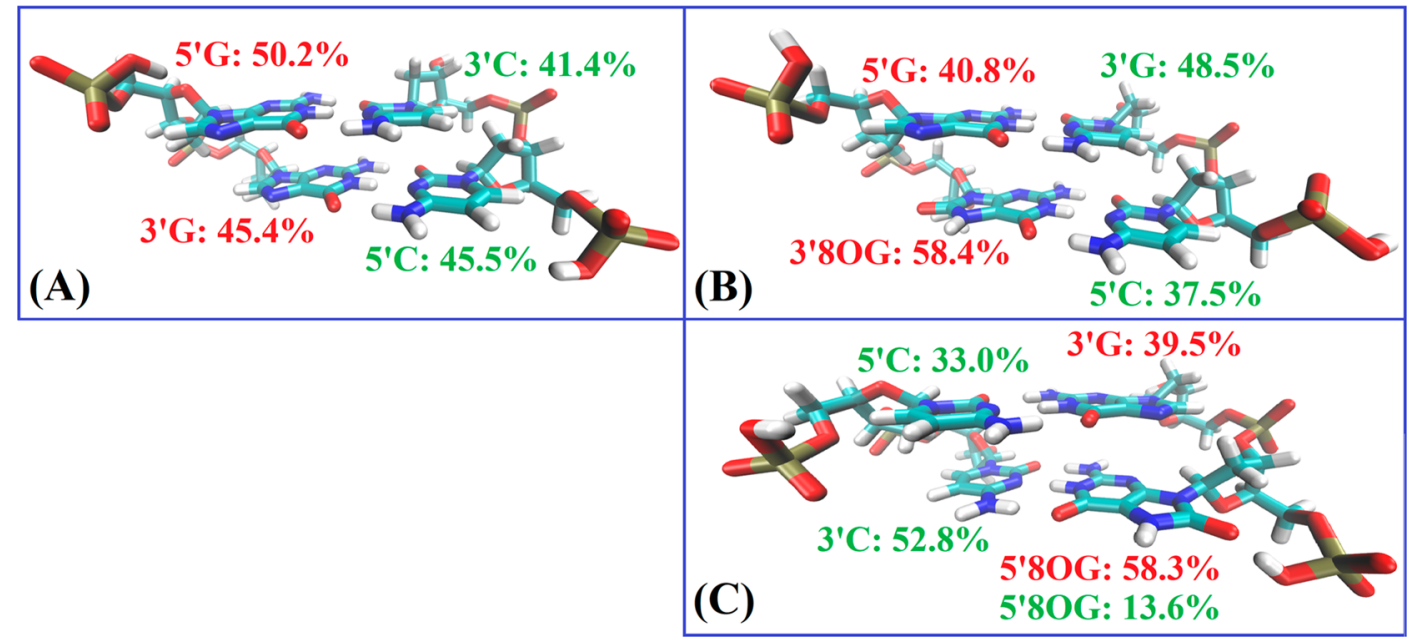

Figure 5. Optimized geometry of the (A) 2 G-C nucleotide pairs, the (B) (G-C)-(8OG-C) nucleotide pairs, and the (C) (8OG-C)-(G-C) nucleotide pairs indicating the system components that concentrate the major fractions of the hole (red) and the excess electron (green). The distributions correspond to vertical IEs/EAs.

Taking the VIEs and VEAs of large base and nucleotide fragments into an overall consideration, the analysis of the hole and excess electron charges yield charge distributions that are consistent with the trends in energy difference observed for native vs defect fragments of the same size. In defect fragments, a major fraction of the hole charge (close to $60 \%$ on 2 -pair systems, and more than $40 \%$ on 3-pair systems) is localized on the $8 \mathrm{OG}$ base. This outcome is line with all defect systems having a lower VIE than their native counterparts. On the other hand, electron distributions are less consistent and demonstrate that the excess charge localization patterns are insensitive to the insertion of 8OG. Such an observation can be anticipated from the very similar VEAs calculated for native and 8OG-containing DNA fragments of the same size.

The hole/excess electron distributions corresponding to AIEs/AEAs of single G-C and 8OG pairs (Tables S17 and S18) are similar to the corresponding VIE/VEA distributions, with the hole and excess electron density being entirely found on the respective purine ( $G$ or $80 G$ ) and the $C$ base, respectively.

The hole distributions corresponding to the AIE of the 2 (GC), (G-C)-(8OG-C), and (8OG-C)-(G-C) fragments (Tables S19-S21) bare again strong similarities to the respective VIE distributions. In the native fragment (Table S19), the hole is again shared between the two G bases (65.5\% and 34.5\%), while in the defect fragments (Tables S20 and S21) the impact of $8 \mathrm{OG}$ is even more pronounced, with the hole density being fully localized on the defect. This picture is consistent with VIE of the defect fragments, with AIE values of the defect that are $0.23 \mathrm{eV}$ lower compared to the native one. On the other hand, in AEA distributions, the excess electron density lies entirely on a single $\mathrm{C}$ base in all three systems composed of two base pairs. These identical distributions between native and defect fragments are fully consistent with the almost identical AEAs (Table 5). Combined with the very small relative difference in AEA between native and defect fragments, this finding further points to a minimal effect of $80 \mathrm{O}$ in the electron capturing properties of DNA, which was also concluded from the analysis of the VEA-related excess electron distributions.

Overall, the findings from both vertical and adiabatic energy gap distributions are in line with the experimental evidence that damaged DNA fragments can act as sinks of oxidative damage but also suggest that a significant enhancement of electron capturing ability is unlikely.

Extent of Delocalization of Oxidative Damage and Effect of the DFT Functional. An experimental study of Sevilla and co-workers ${ }^{86}$ suggested that, in guanine stacks, oxidative damage will predominantly occur on the $5^{\prime} \mathrm{G}$ of the stack. This finding was further supported by quantum calculations. ${ }^{87}$ At a first glance, this finding seems in contradiction with our VIE and AIE-related hole distributions of native fragments, in which the hole is delocalized over the guanines. Nonetheless, the aforementioned experiments were carried out at $77 \mathrm{~K}$, while the theoretical determination of the hole localization pattern was performed for single stranded GG and GGG stacks in the gas phase. These setups do not correspond to the biologically relevant conditions considered here and, consequently, are most probably not directly applicable to our systems.

To further investigate this, the hole (and excess electron) distributions for base pair stacks composed of multiple base pairs were redetermined, using the $\omega \mathrm{B} 97 \mathrm{XD}$ functional for the B3LYP-optimized geometries. The results are shown in detail in Tables S22-S26 of the Supporting Information. The hole distribution obtained for the $2 \mathrm{G}-\mathrm{C}$ pairs (Table S22) is very similar to the one obtained with B3LYP, with exactly $50 \%$ of the hole density residing on each guanine base. For the 3 G-C pairs (Table S25), the hole is also shared between two guanines, with fractions of $52 \%$ and $48 \%$, respectively. Hole distributions obtained with $\omega \mathrm{B} 97 \mathrm{XD}$ on defect fragments (Tables S23, S24, and S26) demonstrate almost complete localization to $8 \mathrm{OG}$, compared to $60 \%$ with B3LYP.

Delocalization of oxidative damage over several DNA bases-including fragments containing guanine-rich sequences-has been observed and reported both experimentally ${ }^{88-90}$ and computationally. ${ }^{91,92}$ In addition to this evidence, given the comparable VIE estimates of B3LYP and $\omega$ B97XD functionals for systems where experimental data are available, it is not a priori possible to determine which functional provides the most accurate description of the hole density distribution. They both correctly predict that the 8OG base is a stronger trap of oxidative damage than $\mathrm{G}$, as in both cases it possesses the largest fraction of the hole density. Given though 
that G also has a low VIE, the possibility that it also captures a smaller fraction of the hole cannot be ruled out.

On a broader perspective, this outcome indicates that, when determining the hole distribution of a defect fragment with different DFT functionals, $80 \mathrm{OG}$ will emerge as the more potent trap, and the extent of delocalization to other $G$ bases will depend on the properties of the functional. A similar behavior can be expected for the excess electron, with complete localization or delocalization over several $\mathrm{C}$ bases, depending on the DFT functional of choice. It is worth linking this analysis with the experimental evidence reported by Schuster et al. ${ }^{88}$ that shows that besides hopping between individual nucleobases, hopping of the hole can also occur between stacks of DNA bases in which the positive charge is successively delocalized. The latter is highly probable in DNA fragments where purine bases are consecutively stacked in the same strand, ${ }^{88}$ which is the case for the systems in the present study.

\section{CONCLUSIONS AND POTENTIAL IMPLICATIONS FOR DNA DAMAGE DETECTION AND REPAIR}

In this work, we have investigated the assumptions at the basis of the repair mechanism of oxidative damages in DNA proposed by Barton et al., ${ }^{5,6}$ by means of static quantum calculations. The stimulus that triggers this mechanism is the presence of an oxidative agent. Given that the PCM-computed VIEs and AIEs for stacks of (G-C) bases or nucleotides are lower than those of single G or G-C pairs, it is highly possible that G-C rich sequences can indeed localize holes, efficiently triggering the repair process. The same can potentially hold for $8 \mathrm{OG}$ containing fragments, with 8-oxoguanine glycosylase as initiator of the process.

VIEs and AIEs of defect fragments were consistently lower than the ones of their native counterparts, with the relative difference being of the order of $0.1 \mathrm{eV}$ for the maximum sizes considered ( 3 base pairs $/ 2$ nucleotide pairs). In addition, an analysis of the corresponding charge distributions also points to an enhancement of the hole capturing properties of damaged DNA compared to native fragments. In line with extensive experimental evidence, ${ }^{16,17,93-95}$ our results demonstrate the ability of 8-oxoguanine to act as a sink of holes migrating through DNA, and thus the potential of 8OGcontaining sequences to indirectly protect other DNA regions from oxidative damage. On the contrary, the computed VEA and AEA values are very similar, with relative differences in the range of $0.01 \mathrm{eV}$, i.e., values that are probably beyond the accuracy of the employed approach, showing no consistent trend as a function of the system size. The corresponding excess electron charge distributions are also in line with this observation indicating that the effect of $80 G$ on DNA's electron capturing properties is very small. These findings suggest that the presence of a DNA-mediated electron transfer mechanism for the detection of defect lesions by BER enzymes is unlikely, as the defect's impact on the DNA's electron capturing efficiency appears to be too small to be exploited. Nonetheless, given the evidence that the hole capturing ability of DNA is enhanced upon replacement of G by $80 G$, a hole transfer-based CT scheme for the recognition of damaged DNA bases may instead be a possibility.

On the other hand, even subtle differences in IEs and EAs between native and damaged DNA might have a significant impact on the redox chemistry of DNA, which could be exploited by the cell in order to identify and repair damaged bases. Further computational work, especially on large DNA fragments simulated in physiological conditions (physiological concentration of counterions and explicit water molecules), might contribute to obtaining a deeper understanding of this important issue. In fact, we have performed some preliminary mixed quantum/classical (QM/MM) simulations of two large DNA fragments, a native and an 8OG-containing one, in biologically relevant conditions. They reveal that VIEs fluctuate considerably, with standard deviations of up to 0.25 $\mathrm{eV}$. Moreover, preliminary estimates of redox potentials reveal an increase of the order of $1 \mathrm{~V}$ when $80 \mathrm{OG}$ is introduced. Considering also the experimentally reported relative change of redox potential at the nucleoside level $(8 \mathrm{OG}>\mathrm{G})^{17}$ of $0.55 \mathrm{~V}$, such a finding highlights the importance that the conformational dynamics of the DNA can have on these properties, when an explicit environment is considered. The fully detailed analysis of these simulations and interpretation of the results will be the subject of a future article that will complement the current work in further assessing the role that redox chemistry has in the recognition and repair of defect bases.

\section{ASSOCIATED CONTENT}

\section{Supporting Information}

The Supporting Information is available free of charge on the ACS Publications website at DOI: 10.1021/acs.jctc.8b00645.

This additional document contains tables with the vertical energy gaps of $\mathrm{G} / 8 \mathrm{OG}$ bases/nucleotides obtained at different levels of theory in the gas phase and with the PCM model. Moreover, the results obtained from the gas phase calculations, as well as from the test setups with $\mathrm{Na}^{+}$ions, are discussed. In addition, detailed hole and excess electron distributions are presented for all systems. The optimized geometries of all systems mentioned in the Computational Methods section are also provided. Finally, cited references with more than 10 authors are presented in full detail (PDF)

\section{AUTHOR INFORMATION}

\section{Corresponding Author}

*(U.R.) E-mail: ursula.roethlisberger@epfl.ch. ORCID

Polydefkis Diamantis: 0000-0003-0535-3158

Ivano Tavernelli: 0000-0001-5690-1981

Ursula Rothlisberger: 0000-0002-1704-8591

\section{Present Address}

${ }^{\dagger}$ (I.T.) IBM-Research, Zurich Research Laboratory, CH-8803 Rüschlikon, Zurich, Switzerland.

\section{Funding}

The research was supported by the Swiss National Science Foundation (NSF, Grants No. 200020-146645 and No. 200020-165863) and the NCCR MUST.

\section{Notes}

The authors declare no competing financial interest.

\section{ACKNOWLEDGMENTS}

The authors thank the DIT/EPFL and the CSCS for computer time.

\section{REFERENCES}

(1) Clancy, S. DNA Damage and Repair: Mechanisms for Maintaining DNA Integrity. Nat. Educ. 2008, 1 (1), 103. 
(2) Odell, I. D.; Wallace, S. S.; Pederson, D. S. Rules of Engagement for Base Excision Repair in Chromatin. J. Cell. Physiol. 2013, 228, 258-266.

(3) Dianov, G. L.; Hübscher, U. Mammalian Base Excision Repair: the Forgotten Archangel. Nucleic Acids Res. 2013, 41, 3483-3490.

(4) Robertson, A. B.; Klungland, A.; Rognes, T.; Leiros, I. Base excision repair: the long and short of it. Cell. Mol. Life Sci. 2009, 66, 981-993.

(5) Yavin, E.; Boal, A. K.; Stemp, E. D. A.; Boon, E. M.; Livingston, A. L.; O'Shea, V. L.; David, S. S.; Barton, J. K. Protein-DNA charge transport: Redox activation of a DNA repair protein by guanine radical. Proc. Natl. Acad. Sci. U. S. A. 2005, 102, 3546-3551.

(6) Boal, A. K.; Genereux, J. C.; Sontz, P. A.; Gralnick, J. A.; Newman, D. K.; Barton, J. K. Redox signaling between DNA repair proteins for efficient lesion detection. Proc. Natl. Acad. Sci. U. S. A. 2009, 106, 15237-15242.

(7) Genereux, J. C.; Boal, A. K.; Barton, J. K. DNA-Mediated Charge Transport in Redox Sensing and Signaling. J. Am. Chem. Soc. 2010, 132, 891-905.

(8) Sontz, P. A.; Muren, N. B.; Barton, J. K. DNA Charge Transport for Sensing and Signaling. Acc. Chem. Res. 2012, 45, 1792-1800.

(9) Grodick, M. A.; Segal, H. M.; Zwang, T. J.; Barton, J. K. DNAMediated Signaling by Proteins with 4Fe-4S Clusters Is Necessary for Genomic Integrity. J. Am. Chem. Soc. 2014, 136, 6470-6478.

(10) O’Brien, E.; Silva, R. M. B.; Barton, J. K. Redox Signaling through DNA. Isr. J. Chem. 2016, 56, 705-723.

(11) Markus, T. Z.; Daube, S. S.; Naaman, R. Cooperative Effect in the Electronic Properties of Human Telomere Sequence. J. Phys. Chem. B 2010, 114, 13897-13903.

(12) Markus, T. Z.; Daube, S. S.; Naaman, R.; Fleming, A. M.; Muller, J. G.; Burrows, C. J. Electronic Structure of DNA - Unique Properties of 8-Oxoguanosine. J. Am. Chem. Soc. 2009, 131, 89-95.

(13) Fujitsuka, M.; Majima, T. Hole and Excess Electron Tranfer Dynamics in DNA. Phys. Chem. Chem. Phys. 2012, 14, 11234-11244.

(14) Eriksen, K. A. Location of DNA damage by charge exchanging repair enzymes: Effect of cooperativity on location time. Theor. Biol. Med. Modell. 2005, 2, 15.

(15) Heller, A. On the hypothesis of cathodic protection of genes. Faraday Discuss. 2000, 116, 1-13.

(16) Gasper, S. M.; Schuster, G. B. Intramolecular Photoinduced Electron Transfer to Anthraquinones Linked to Duplex DNA: The Effect of Gaps and Traps on Long-Range Radical Cation Migration. J. Am. Chem. Soc. 1997, 119, 12762-12771.

(17) Steenken, S.; Jovanovic, S. V.; Bietti, M.; Bernhard, K. The Trap Depth (in DNA) of 8-Oxo-7.8-dihydro-2' deoxyguanosine as Derived from Electron-Transfer Equilibria in Aqueous Solution. J. Am. Chem. Soc. 2000, 122, 2373-2374.

(18) Hush, N. S.; Cheung, A. S. Ionization Potentials and Donor Properties of Nucleic Acid Bases and Related Compounds. Chem. Phys. Lett. 1975, 34, 11-13.

(19) Dougherty, D.; Younathan, E. S.; Voll, R.; Abdulnur, S.; McGlynn, S. P. Photoelectron Spectroscopy of Some Biological Molecules. J. Electron Spectrosc. Relat. Phenom. 1978, 13, 379-393.

(20) Belau, L.; Wilson, K. R.; Leone, S. R.; Ahmed, M. VacuumUltraviolet Photoionization Studies of the Microhydration of DNA Bases. J. Phys. Chem. A 2007, 111, 7562-7568.

(21) Zhou, J.; Kostko, O.; Nicolas, C.; Tang, X.; Belau, L.; de Vries, M. S.; Ahmed, M. Experimental Observation of Guanine Tautomers with VUV Photoionization. J. Phys. Chem. A 2009, 113, 4829-4832.

(22) Roca-Sanjuán, D.; Rubio, M.; Merchán, M.; Serrano-Andrés, L. $A b$ initio determination of the ionization potentials of DNA and RNA nucleobases. J. Chem. Phys. 2006, 125, No. 084302.

(23) Li, X.; Sevilla, M. D. DFT Treatment of Radiation Produced Radicals in DNA Model Systems. Adv. Quantum Chem. 2007, 52, 5987.

(24) Cauët, E.; Liévin, J. Radical Cations of the Nucleic Bases and Radiation Damage to DNA: Ab Initio Study. Adv. Quantum Chem. 2007, 52, 121-147.
(25) Paukku, Y.; Hill, G. Theoretical Determination of One-Electron Redox Potentials for DNA Bases, Base Pairs, and Stacks. J. Phys. Chem. A 2011, 115, 4804-4810.

(26) Pluharova, E.; Jungwirth, P.; Bradforth, S. E.; Slavicek, P. Ionization of Purine Tautomers in Nucleobases, Nucleosides, and Nucleotides: From the Gas Phase to the Aqueous Environment. J. Phys. Chem. B 2011, 115, 1294-1305.

(27) Slavicek, P.; Winter, B.; Faubel, M.; Bradforth, S. E.; Jungwirth, P. Ionization of Energies of Aqueous Nucleic Acids: Photoelectron Spectroscopy of Pyrimidine Nucleosides and ab Initio Calculations. J. Am. Chem. Soc. 2009, 131, 6460-6467.

(28) Pluharova, E.; Schroeder, C.; Seidel, R.; Bradforth, S. E.; Winter, B.; Faubel, M.; Slavicek, P.; Jungwirth, P. Unexpectedly Small Effect of the DNA Environment on Vertical Ionization Energies of Aqueous Nucleobases. J. Phys. Chem. Lett. 2013, 4, 3766-3769.

(29) Sugiyama, H.; Saito, I. Theoretical Studies of GG-Specific Photocleavage of DNA via Electron Transfer: Significant Lowering of Ionization Potential and 5'-Localization of HOMO of Stacked GG Bases in B-Form DNA. J. Am. Chem. Soc. 1996, 118, 7063-7068.

(30) Yokojima, S.; Yoshiki, N.; Yanoi, W.; Okada, A. Solvent Effects on Ionization Potentials of Guanine Runs and Chemically Modified Guanine in Duplex DNA: Effect of Electrostatic Interaction and Its Reduction due to Solvent. J. Phys. Chem. B 2009, 113, 16384-16392.

(31) Rooman, M.; Wintjens, R. Sequence and conformation effects on ionization potential and charge distribution of homo-nucleobase stacks using M06-2X hybrid density functional theory calculations. J. Biomol. Struct. Dyn. 2014, 32, 532-545.

(32) Svozil, D.; Jungwirth, P.; Havlas, Z. Electron Binding to Nucleic Acid Bases. Experimental and Theoretical Studies. A Review. Collect. Czech. Chem. Commun. 2004, 69, 1395-1428.

(33) Dutta, A. K.; Sengupta, T.; Vaval, N.; Pal, S. Electron Attachment to DNA and RNA Nucleobases: AN EOMCC Investigation. Int. J. Quantum Chem. 2015, 115, 753-764.

(34) Aflatooni, K.; Gallup, G. A.; Burrow, P. D. Electron Attachment Energies of the DNA Bases. J. Phys. Chem. A 1998, 102, 6205-6207.

(35) Chen, E. S. D.; Chen, E. C. M.; Sane, N.; Shulze, S. Classification of organic molecules to obtain electron affinities from half-wave reduction potentials: cytosine, uracil, thymine, guanine and adenine. Bioelectrochem. Bioenerg. 1999, 48, 69-78.

(36) Periquet, V.; Moreau, A.; Carles, S.; Schermann, J. P.; Desfrancois, C. Cluster size effects upon anion solvation of $\mathrm{N}$ heterocyclic molecules and nucleic acid bases. J. Electron Spectrosc. Relat. Phenom. 2000, 106, 141-151.

(37) Harinipriya, S.; Sangaranarayanan, M. V. Estimation of the electron affinities of nucleobases using thermochemical data and structural considerations. J. Mol. Struct. 2003, 644, 133-138.

(38) Linstrom, P. J., Mallard, W. G., Eds.; NIST Chemistry WebBook, NIST Standard Reference Database Number 69; National Institute of Standards and Technology: Gaithersurg, MD; http://webbook.nist. gov.

(39) Sevilla, M. D.; Besler, B.; Colson, A.-O. Ab Initio Molecular Orbital Calculations of DNA Radical Ions. 5. Scaling of Calculated Electron Affinities and Ionization Potentials to Experimental Values. J. Phys. Chem. 1995, 99, 1060-1063.

(40) Russo, N.; Toscano, M.; Grand, A. Theoretical Determination of Electron Affinity and Ionization Potential of DNA and RNA Bases. J. Comput. Chem. 2000, 21, 1243-1250.

(41) Wesolowski, S. S.; Leininger, M. L.; Pentchev, P. N.; Schaefer, H. F., III Electron Affinities of the DNA and RNA Bases. J. Am. Chem. Soc. 2001, 123, 4023-4028.

(42) Li, X.; Cai, Z.; Sevilla, M. D. DFT Calculations of the Electron Affinities of Nucleid Acid Bases: Dealing with Negative Electron Affinities. J. Phys. Chem. A 2002, 106, 1596-1603.

(43) Richardson, N. A.; Gu, J.; Wang, S.; Xie, Y.; Schaefer, H. F., III DNA Nucleosides and Their Radical Anions: Molecular Structures and Electron Affinities. J. Am. Chem. Soc. 2004, 126, 4404-4411.

(44) Sarmah, P.; Deka, R. C. Density functional studies on the electron affinities of DNA and RNA bases. Mol. Simul. 2008, 34, 879885 . 
(45) Nguyen, N. L.; Borghi, G.; Ferretti, A.; Marzari, N. FirstPrinciples Photoemission Spectroscopy of DNA and RNA Nucleobases from Koopmans-Compliant Functionals. J. Chem. Theory Comput. 2016, 12, 3948-3958.

(46) Roca-Sanjuán, D.; Merchán, M.; Serrano-Andrés, L.; Rubio, M. $\mathrm{Ab}$ initio determination of the electron affinities of DNA and RNA nucleobases. J. Chem. Phys. 2008, 129, No. 095104.

(47) Jalbout, A. F.; Adamowicz, L. Electron Attachment to Base Complexes. Adv. Quantum Chem. 2007, 52, 231-251.

(48) Gu, J.; Leszczynski; Schaefer, H. F., III Interactions of Electrons with Bare and Hydrated Biomolecules: From Nucleic Acid Bases to DNA Segments. Chem. Rev. 2012, 112, 5603-5640.

(49) Colson, A. O.; Besler, B.; Sevilla, M. D. Ab Initio Molecular Orbital Calculations on DNA Radical Ions. 4. Effect of Hydration on Electron Affinities and Ionisation Potentials of Base Pairs. J. Phys. Chem. 1993, 97, 13852-13859.

(50) Sevilla, M. D.; Besler, B.; Colson, A. O. Ab Initio Molecular Orbital Calculations on DNA Radical Ions. Electron Attachment to Uracil. Theoretical ab Initio Study. J. Phys. Chem. 1994, 98, 2215.

(51) Gu, J.; Xie, Y.; Schaefer, H. F. Electron attachment induced proton transfer in a DNA nucleoside pair: 2'-deoxyguanosine-2'deoxycytidine. J. Chem. Phys. 2007, 127, 155107.

(52) Li, X.; Cai, Z.; Sevilla, M. D. Energetics of the Radical Ions of the AT and AU Base Pairs: A Density Functional Theory (DFT) Study. J. Phys. Chem. A 2002, 106, 9345-9351.

(53) Gu, J.; Wong, N.-B.; Xie, Y.; Schaefer, H. F., III Electron Attachment to a Hydrated DNA Duplex: The Dinucleoside Phosphate Deoxyguanylyl-3',5'-Deoxycytidine. Chem. - Eur. J. 2010, $16,13155-13162$.

(54) Gu, J.; Xie, Y.; Schaefer, H. F., III Electron Attachment to Hydrated Oligonucleotide Dimers: Guanylyl-3',5'-Cytidine and Cytidylyl-3',5'-Guanosine. Chem. - Eur. J. 2010, 16, 5089-5096.

(55) Gu, J.; Wang, J.; Leszczynski, J. Electron Attachment to the Cytosine-Centered DNA Single Strands: Does Base Stacking Matter? J. Phys. Chem. B 2012, 116, 1458-1466.

(56) Sun, H.; Zhang, S.; Sun, Z. Applicability of optimal functional tuning in density functional calculations of ionization potentials and electron affinities of adenine-thymine nucleobase pairs and clusters. Phys. Chem. Chem. Phys. 2015, 17, 4337-4345.

(57) Palivec, V.; Pluharova, E.; Unger, I.; Winter, B.; Jungwirth, P. DNA Lesion Can Facilitate Base Ionization: Vertical Ionization Energies of Aqueous 8-Oxoguanine and its Nucleoside and Nucleotide. J. Phys. Chem. B 2014, 118, 13833-13837.

(58) Prat, F.; Houk, K. N.; Foote, C. S. Effect of Guanine Stacking on the Oxidation of 8-Oxoguanine in DNA. J. Am. Chem. Soc. 1998, 120, 845-846.

(59) Pacheco-Ortín, S.; Gaitán Lozano, R.; Agacino Valdés, E. Possible DNA Damage by Oxidation Products of Guanine: A Density Functional and Electron Propagator Theoretical Study. Int. J. Quantum Chem. 2012, 112, 2840-2847.

(60) Frisch, M. J.; Trucks, G. W.; Schlegel, H. B.; Scuseria, G. E.; Robb, M. A.; Cheeseman, J. R.; Scalmani, G.; Barone, V.; Mennucci, B.; Petersson, G. A.; et al.. Gaussian 09, Revision A.1; Gaussian, Inc.: Wallingford, CT, U.S.A., 2009.

(61) Kohn, W.; Sham, L. J. Self-Consistent Equations Including Exchange and Correlation Effects. Phys. Rev. 1965, 140, A1133A1138.

(62) Burke, K.; Wagner, L. O. DFT in a Nutshell. Int. J. Quantum Chem. 2013, 113, 96-101.

(63) Miertus, S.; Scrocco, E.; Tomasi, J. Electrostatic Interaction of a Solute with the Continuum. A Direct Utilization of $\mathrm{Ab}$ Initio Molecular Potentials For The Prevision of Solvent Effects. Chem. Phys. 1981, 55, 117-129.

(64) Amovilli, C.; Barone, V.; Cammi, R.; Cances, E.; Cossi, M.; Mennucci, B.; Pomelli, C. S.; Tomasi, J. Recent Advances in the Description of Solvent Effects with the Polarizable Continuum Model. Adv. Quantum Chem. 1998, 32, 227-261.
(65) Tomasi, J.; Cammi, R.; Mennucci, B.; Cappelli, C.; Corni, S. Molecular properties in solution described with a continuum solvation model. Phys. Chem. Chem. Phys. 2002, 4, 5697-5712.

(66) Becke, A. D. Density-functional exchange-energy approximation with correct asymptotic behavior. Phys. Rev. A: At., Mol., Opt. Phys. 1988, 38, 3098-3100.

(67) Lee, C.; Yang, W.; Parr, R. G. Development of the ColleSalvetti correlation-energy formula into a functional of the electron density. Phys. Rev. B: Condens. Matter Mater. Phys. 1988, 37, 785-789.

(68) Godbout, N.; Salahub, D. R.; Andzelm, J.; Wimmer, E. Optimization of Gaussian-type basis sets for local spin density functional calculations. Part I. Boron through neon, optimization technique and validation. Can. J. Chem. 1992, 70, 560-571.

(69) Becke, A. D. Density-functional thermochemistry. III. The role of exact exchange. J. Chem. Phys. 1993, 98, 5648-5652.

(70) Zhao, Y.; Truhlar, D. G. The M06 suite of density functionals for main group thermochemistry, thermochemical kinetics, noncovalent interactions, excited states, and transition elements: two new functionals and systematic testing of four M06-class functionals and 12 other functionals. Theor. Chem. Acc. 2008, 120, 215-241.

(71) Hehre, W. J.; Ditchfield, R.; Pople, J. A. Self-Consistent Molecular Orbital Methods. XII. Further Extensions of GaussianType Basis Sets for Use in Molecular Orbital Studies of Organic Molecules. J. Chem. Phys. 1972, 56, 2257-2261.

(72) Dill, J. D.; Pople, J. A. Self-consistent molecular orbital methods. XV. Extended Gaussian-type basis sets for lithium, beryllium, and boron. J. Chem. Phys. 1975, 62, 2921-2923.

(73) Francl, M. M.; Pietro, W. J.; Hehre, W. J.; Binkley, J. S.; Gordon, M. S.; DeFrees, D. J.; Pople, J. A. Pople, Selfconsistent molecular orbital methods. XXIII. A polarization type basis set for second row elements J. A. J. Chem. Phys. 1982, 77, 3654-3665.

(74) Clark, T.; Chandrasekhar, J.; Schleyer, P. v. R.; Spitznagel, G. W. Efficient Diffuse Function- Augmented Basis Sets for Anion Calculations. III.* The 3-21+G Basis Set for First-Row Elements, LiF. J. Comput. Chem. 1983, 4, 294-301.

(75) Krishnan, R.; Binkley, J. S.; Seeger, R.; Pople, J. A. Selfconsistent molecular orbital methods. XX. A basis set for correlated wave functions. J. Chem. Phys. 1980, 72, 650-654.

(76) Gill, P. M. W.; Johnson, B. G.; Pople, J. A.; Frisch, M. J. The performance of the Becke-Lee-Yang-Parr (B-LYP) density functional theory with various basis sets. Chem. Phys. Lett. 1992, 197, 499-505.

(77) Kumar, A.; Sevilla, M. D. Proton Transfer Induced SOMO-toHOMO Level Switching in One-Electron Oxidized A-T and G-C Base Pairs: A Density Functional Theory Study. J. Phys. Chem. B 2014, 118, $5453-5458$

(78) Chai, J.-D.; Head-Gordon, M. Systematic optimization of longrange corrected hybrid density functionals. J. Chem. Phys. 2008, 128, No. 084106.

(79) Chai, J.-D.; Head-Gordon, M. Long-range corrected hybrid density functionals with damped atom-atom dispersion corrections. Phys. Chem. Chem. Phys. 2008, 10, 6615-6620.

(80) Cossi, M.; Barone, V. Solvent Effect on Vertical Electronic Transitions by the Polarizable Continuum Model. J. Chem. Phys. 2000, 112, 2427-2435.

(81) Cossi, M.; Barone, V. Separation Between Fast and Slow Polarizations in Continuum Solvation Models. J. Phys. Chem. A 2000, 104, 10614-10622.

(82) Jagoda-Cwiklik, B.; Slavíček, P.; Cwiklik, L.; Nolting, D.; Winter, B.; Jungwirth, P. Ionization of Imidazole in the Gas Phase, Microhydrated Environments, and in Aqueous Solution. J. Phys. Chem. A 2008, 112, 3499-3505.

(83) Jagoda-Cwiklik, B.; Slavíček, P.; Nolting, D.; Winter, B.; Jungwirth, P. Ionization of Aqueous Cations: Photoelectron Spectroscopy and ab Initio Calculations of Protonated Imidazole. J. Phys. Chem. B 2008, 112, 7355-7358.

(84) Pluharova, E.; Slavicek, P.; Jungwirth, P. Modeling Photoionization of Aqueous DNA and Its Components. Acc. Chem. Res. 2015, 48, 1209-1217. 
(85) Yang, X.; Wang, X. B.; Vorpagel, E. R.; Wang, L. S. Direct experimental observation of the low ionization potentials of guanine in free oligonucleotides by using photoelectron spectroscopy. Proc. Natl. Acad. Sci. U. S. A. 2004, 101, 17588-17592.

(86) Adhikary, A.; Khanduri, D.; Sevilla, M. D. Direct Observation of Hole Protonation State and Hole Localization Site in DNAOligomers. J. Am. Chem. Soc. 2009, 131, 8614-8619.

(87) Kumar, A.; Sevilla, M. D. Density Functional Theory Studies of Extent of Hole Delocalization in One-Electron Oxidized Adenine and Guanine Base Stacks. J. Phys. Chem. B 2011, 115, 4990-5000.

(88) Liu, C. S.; Schuster, G. B. Base Sequence Effects in Radical Cation Migration in Duplex DNA: Support for the Polaron-Like Hopping Model. J. Am. Chem. Soc. 2003, 125, 6098-6102.

(89) Shao, F.; O’Neill, M. A.; Barton, J. K. Long-range oxidative damage to cytosines in duplex DNA. Proc. Natl. Acad. Sci. U. S. A. 2004, 101, 17914-17919.

(90) Capobianco, A.; Caruso, T.; D’Ursi, A. M.; Fusco, S.; Masi, A.; Scrima, M.; Chatgilialoglu, C.; Peluso, A. Delocalized Hole Domains in Guanine-Rich DNA Oligonucleotides. J. Phys. Chem. B 2015, 119, 5462-5466.

(91) Barnett, R. N.; Cleveland, C. L.; Joy, A.; Landman, U.; Schuster, G. B. Charge Migration in DNA: Ion-Gated Transport. Science 2001, 294, 567-571.

(92) Wu, J.; Walker, V. E. J.; Boyd, R. J. Theoretical Study of Polaron Formation in Poly(G)-Poly(C) Cations. J. Phys. Chem. B 2011, 115, 3136-3145.

(93) Hickerson, R. P.; Prat, F.; Muller, J. G.; Foote, C. S.; Burrows, C. J. Sequence and Stacking Dependence of 8-Oxoguanine Oxidation: Comparison of One-Electron vs Singlet Oxygen Mechanisms. J. Am. Chem. Soc. 1999, 121, 9423-9428.

(94) Giese, B.; Biland, A. Recent developments of charge injection and charge transfer in DNA. Chem. Commun. 2002, 18, 667-672.

(95) Shukla, L. I.; Adhikary, A.; Pazdro, R.; Sevilla, M. D. Formation of 8-oxo-7,8-dihydroguanine-radicals in $\gamma$-irradiated DNA by multiple one-electron oxidations. Nucleic Acids Res. 2004, 32, 6565-6574. 